\title{
The aftermath of a perioperative death: who cares for the clinician?
}

Working in the perioperative environment entails exposure to traumatic and sometimes catastrophic events such as a perioperative death (PD). PD can be a uniquely devastating experience, and has the potential to lead to long-term negative physical and psychological effects for the staff involved, especially when appropriate support is absent. ${ }^{12}$ In a number of practice settings, these destabilizing effects have been shown to detrimentally compromise individual and team performance. ${ }^{3}$ This is of particular concern in the perioperative setting, since deterioration of individual competence and subsequent team performance has been directly linked to poor patient outcomes. ${ }^{45}$ Despite numerous studies establishing this link, there has been little research exploring clinicians' experiences of PD and organisational support for front-line clinicians remains alarmingly inconsistent. The question remains, who

\footnotetext{
${ }^{1}$ Gazoni FM, Durieux ME, Wells L. Life after death: the aftermath of perioperative catastrophes. Anesth Analg 2008; 107(2): 591-600.

${ }^{2}$ Pratt SD, Jachna BR. Care of the clinician after an adverse event. Int J of Obstet Anesth 2015; 24(1):54-63.

${ }^{3}$ Seys D, Scott S, Wu A, et al. Supporting involved health care professionals (second victims) following an adverse health event: a literature review. Int J Nurs Stud 2013; 50(5): 678-87.

${ }^{4}$ Awad SS, Fagan SP, Bellows C, et al. Bridging the communication gap in the operating room with medical team training. Am J Surg 2005; 190(5): 770-4.

${ }^{5}$ Mazzocco K, Petitti DB, Fong KT, et al. Surgical team behaviors and patient outcomes. Am J Surg 2009; 197(5): 678-85.
} 
is responsible to the clinician in the aftermath of a perioperative death?

A PD describes the death of a patient occurring throughout the perioperative period, after their arrival in the anaesthetic room and before leaving the post-anaesthesia care unit. ${ }^{6} \mathrm{PD}$ has been identified as an international issue; in 2009, the World Health Organization flagged surgical death as a patient safety issue, bringing to light the growing prevalence of surgery and its associated risks and complications worldwide. ${ }^{7}$ In the United Kingdom (UK) there are a subset of high-risk patients that make up $10 \%$ of surgical patients but whom account for more than $80 \%$ of post-operative deaths. ${ }^{8}$ This group of patients are more likely to be anaesthetised and operated on at specialist tertiary centres, and staff working there are more likely to be exposed to PD. Despite this prevalence, the small body of research exploring the effects of PD on staff has focused primarily on surgeons and anaesthetists. ${ }^{9}{ }^{10}$ Until recently there has been little interest in exploring the impact of PD on other healthcare professionals

\footnotetext{
${ }^{6}$ Rodger, D, Atwal, A. How to mitigate the effects of peri-operative death on nursing staff. Nursing Times 2018; 114(8): 26-29.

${ }^{7}$ World Health Organization [Internet]. World Health Organization guidelines for safe surgery safe surgery saves lives; 2009. Available from: http://apps.who.int/iris/bitstream/handle/10665/44185/9789241598552_eng.pdf?sequence=1.

${ }^{8}$ Pearse RM, Moreno RP, Bauer P, et al. Mortality after surgery in Europe: a 7 day cohort study. Lancet 2012; 380(9847): 1059-1065.

${ }^{9}$ Goldstone AR, Callaghan CJ, Mackay J, et al. Should surgeons take a break after an intraoperative death? Attitude survey and outcome evaluation. BMJ 2004; 328(7436): 379.

${ }^{10}$ Todesco J, Rasic NF, Capstick J. The effect of unanticipated perioperative death on anesthesiologists. Can J Anaesth 2010; 57(4): 361-367.
} 
such as nurses.

Being involved in a PD has been identified as a destabilising experience that can cause the onset of symptoms such as insomnia, depression, flashbacks, anxiety, guilt, shame, loss of empathy and substance abuse. ${ }^{11}{ }^{12}$ In some cases, this phenomenon is best described as a 'second victim' experience. A second victim experience can be broadly understood as the physical and psychological distress triggered by a traumatic event or medical error involving a patient (the primary victim). ${ }^{13} 14$ Left unacknowledged, these negative effects culminate and can become long lasting, jeopardising a professional's competence, confidence and personal relationships, and hindering their ability to flourish in their professional and personal life. ${ }^{11} 12$

Perioperative staff are particularly vulnerable to second victimization following a PD. The care intention underpinning surgery — a philosophy of saving lives executed through sometimes violent interventions_-simultaneous establishes and complicates staffs' identity as

\footnotetext{
${ }^{11}$ Gazoni FM, Amato PE, Malik ZM, et al. The Impact of Perioperative Catastrophes on Anesthesiologists: Results of a National Survey. Anesth Analg 2012; 114(3): 596-603.

${ }^{12}$ Martin TW, Roy RC. Cause for Pause After a Perioperative Catastrophe:

One, Two, or Three Victims? Anesth Analg 2012; 114(3): 485-487.

${ }^{13} \mathrm{Wu} \mathrm{AW}$. Medical error: the second victim: the doctor who makes the mistake needs help too. $B M J$ 2000; 320(7237): 726-727.

${ }^{14}$ Scott SD, Hahn-Cover K (2014) Second Victim. In: Agrawal A (eds) Patient Safety: A Case-Based Comprehensive Guide. New York: Springer.
} 
victims. ${ }^{15} \mathrm{PD}$ throws into question their professional purpose, instigating questions of responsibility, fault, and competence. In addition, the biomedical underpinnings of perioperative culture actually create a framework for victimization by socializing clinicians to disconnect from and often ignore their experiences and emotions following a PD. ${ }^{15}$ The "death denying" culture of the perioperative setting is evidenced in the scarcity of research aimed at understanding and recognizing the experience of 'second victims' in surgical contexts. ${ }^{16}$

We are not suggesting that staff will inevitably become 'second victims' following a PD, as this will be contingent on a number of salient factors. Some professionals will have developed a degree of resilience that protects them from such responses, but this is far from being universal. Michael Traynor has recently identified flaws in the individualistic notion of resilience, highlighting that it provides space for the maintenance of the status quo by making the individual feel responsible for [the trauma they sustain from] organisational failures. ${ }^{17}$ This also entails that professionals are to blame if they are unable to physically or psychologically 'bounce back' from events that may have been outside of their control. Organisations are able to get away with the provision of inadequate support, placing the

\footnotetext{
${ }^{15}$ Hartley H, Wright DK, Vanderspank-Wright B, et al. Dead on the table: a theoretical expansion of the vicarious trauma that operating room clinicians experience when their patients die. Death studies 2018; doi: 10.1080/07481187.2018.1461711

${ }^{16}$ Zimmermann C. Denial of impending death: a discourse analysis of the palliative care literature. Soc Sci Med 2004; 59(8): 1769-80.

${ }^{17}$ Traynor M. Guest editorial: What's wrong with resilience. J Res Nurs 2018; 23(1): 5-8.
} 
impetus on the individual and never addressing the underlying systemic problem(s).

Nevertheless, when adequate support is missing, even the most resilient staff members will be unable to cope following continuous exposure to traumatic events. ${ }^{18}$

It is crucial to note that due to complex interprofessional dynamics and role variability, clinicians can react to a PD very differently and therefore benefit from different resources. Existing research has demonstrated that there is little consistency in the organisational responses, and whilst some staff are provided with adequate support; others encounter an absence and continue to suffer. ${ }^{19}{ }^{21}$ Gaining a better understanding of these phenomena in the perioperative context will help develop effective support aimed at addressing the destabilizing effects of PD, and supporting the multidimensional roles embedded in theatre teams.

Further research in this area is vital for two primary reasons. Firstly, we believe it is morally irresponsible to ignore physical and psychological distress and demonstrates a lack of care and organisational support towards the wellbeing of staff. Secondly, professionals who have

\footnotetext{
${ }^{18}$ Grant L, Kinman G. Emotional Resilience in the Helping Professions and how it can be Enhanced. Health and Social Care Education 2014; 3(1): 23-34.

${ }^{19}$ Michael R, Jenkins HJ. Work-related trauma: the experiences of perioperative nurses. Collegian 2001; 8(1): 19-25.

${ }^{20}$ Breadon R, McColgan K. Perioperative death: a critical reflection-on-action. J Perioper Pract 2012; 22(1): 10-13.

${ }^{21}$ Jithoo S, Sommerville TE. Death on the table: anaesthetic registrars' experiences of perioperative death. Southern African Journal of Anaesthesia and Analgesia 2017; 23(1): 1-5.
} 
become second victims may not be able to participate effectively within the multidisciplinary team, which can have a detrimental effect on patient care and outcomes. ${ }^{34}$

It is imperative that organisations explore the implementation of standardised evidence-based policies that ensure all perioperative staff have access to appropriate support in the aftermath of a PD. Organisations who are failing to do so may be compounding the physical and psychological health of their perioperative staff, in some cases—compromising patient care. 